\title{
Process Considerations in 3D Hand Anthropometric Data Collection
}

\author{
Linsey GRIFFIN ${ }^{\star 1}$, Susan L. SOKOLOWSKI ${ }^{2}$, Emily SEIFERT ${ }^{1}$ \\ ${ }^{1}$ University of Minnesota, St. Paul, (MN), USA \\ ${ }^{2}$ University of Oregon, Portland (OR), USA
}

DOI: $10.15221 / 18.123$ http://dx.doi.org/10.15221/18.123

\begin{abstract}
Traditional hand anthropometric studies are missing several key measurements that are important to designing products and tools for the hand. Specific anthropometric hand data important for hand product design such as gloves include finger lengths, crotch depths, palm and padding, back of hand, and wrist opening; these measurements can improve dexterity, gripping, hand entry, adduction, abduction, squeezing, etc. in the design. The purpose of this paper was to develop a process and special considerations for 3D hand scanning that could help guide future researchers when conducting more robust 3D anthropometric studies for the hand, as related to product design.
\end{abstract}

Over the course of two years, the authors of this paper have developed and refined a process considerations model for 3D hand scanning. The model was developed based on three previous 3D hand scanning studies and over 200 subjects' hand scans. The process considers the subject and population, the 3D technology, landmark methods, hand scanning positions, the scanning research design, scan analysis, and methods of hand-product visualization using 3D hand data.

As technology improves, our processes for collecting data need to adapt. New 3D scanning technology enables a more robust collection of anthropometric, ergonomic, and design data for the hand. Future 3D hand anthropometric data and design research will have a profound impact on future glove and tool design for a range of fields and consumers. The application of the 3D hand scanning process considerations model will enable innovative anthropometric and ergonomic research for the hand to occur, and will ensure the collection of accurate and reliable 3D hand data.

Keywords: Hands, Anthropometrics, 3D Hand Scanning

\section{Introduction}

While hand anthropometric data is available to researchers, it has typically been limited to three basic measurements: hand length, breadth, and circumference (Figure 1) [1]. Relying only on these three measures, can result in mis-proportioned and performing products (Figure 2). When users rely on hand products that are informed from minimal data, it can put the user in danger, as their dexterity and grip are compromised. There is a great need to know more about the 3D form and measures of the entire hand, including how the anthropometry varies in dynamic positions. 3D hand scan data offers designers and engineers endless opportunities to improve not only the design of products, but also the process by which those products are designed.

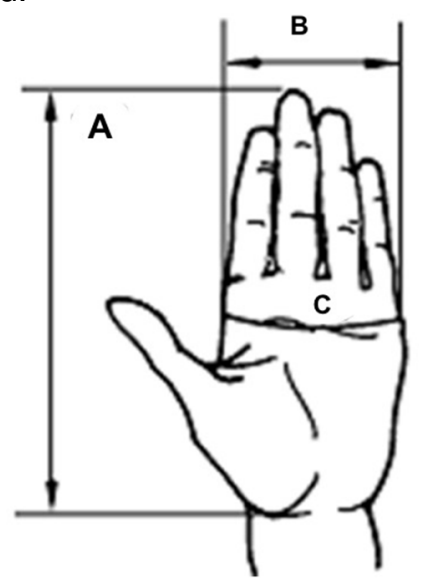

Figure 1. Common hand anthropometric measurements: A) hand length, B) breadth, and C) circumference.

*Igriffin@umn.edu; +1-612- 301-1351; http://wearable.design.umn.edu/ 

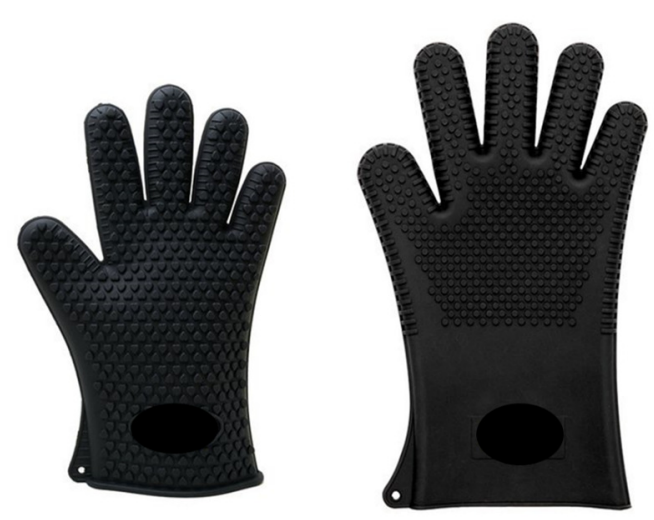

Figure 2. Currently manufactured protective gloves in Medium (left) and X-Large (right) sizes demonstrating how minimal measurements can cause mis-proportioned products.

The 3D scanning equipment landscape has expanded dramatically over the past ten years. New developments provide researchers with hand-held and portable capabilities that are affordable, and can accurately capture the form and dimensions of the hands [2]. New technology advancements also lend to the examination of the hand across a range of postures $[3,4]$. As the market and application for 3D hand scanning has improved, there is a need to develop methodology that produces a repeatable process and reliability data for product creation.

The purpose of this paper was to develop a process and considerations for 3D hand scanning that could help guide future researchers when conducting more robust 3D anthropometric studies for the hand, as related to product design.

\section{Methodology}

Over the course of two years, the authors of this paper have developed and refined a process and considerations model for 3D hand scanning for product and tool design. Over 200 subjects hands have been scanned through three anthropometric studies [4][5][6]. The researchers developed methods to ensure repeatability and reliability of the data.

\section{Results}

A process and considerations model was developed based on three previous 3D hand scanning studies (see Figure 3). The process considers the subject and population, the 3D technology, landmark methods, hand scanning positions, the scanning research design, scan analysis, and methods of handproduct visualization using 3D hand data. Each of these steps is discussed in detail in this section. 


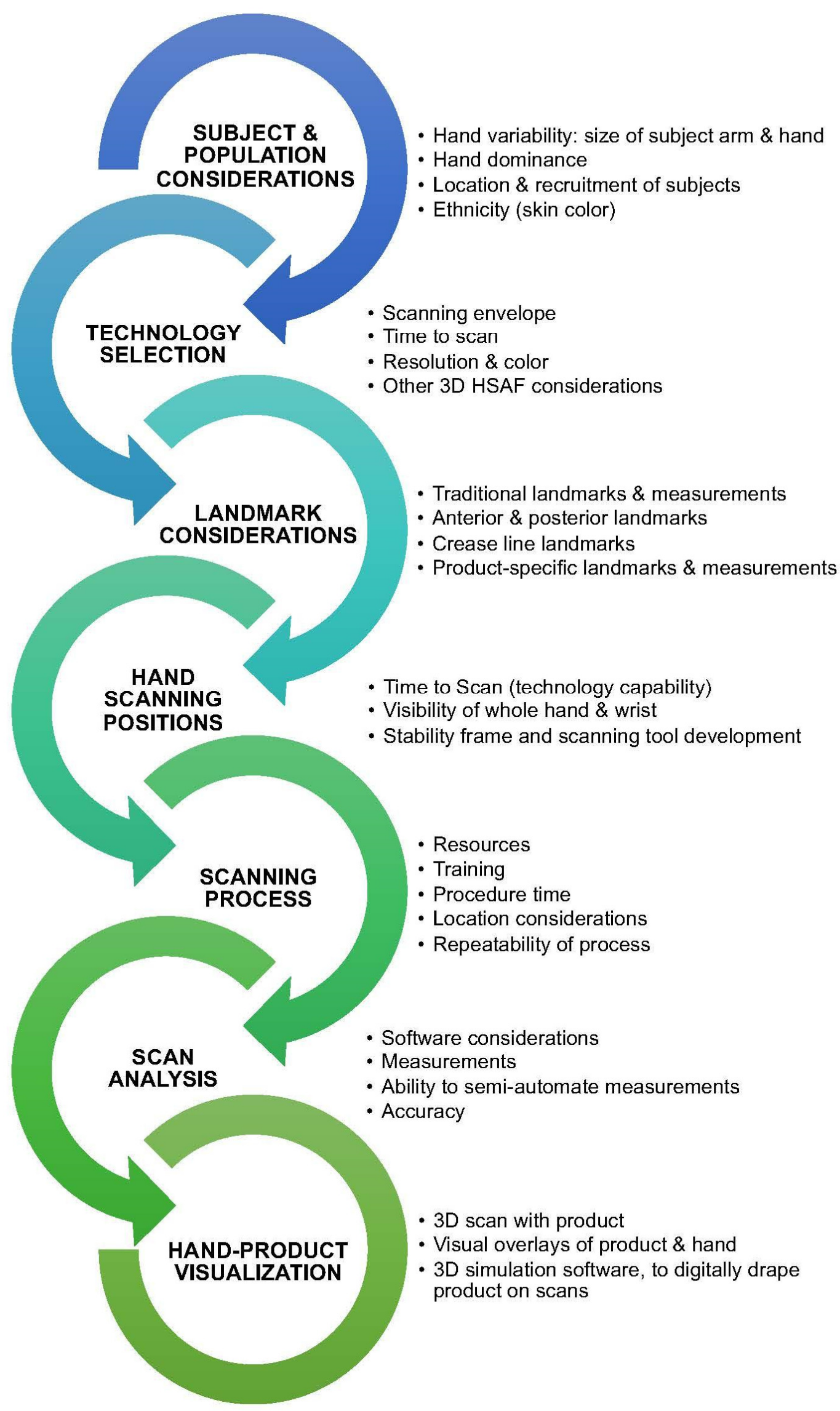

Figure 3. 3D hand scanning process considerations. 


\subsection{Subject and population considerations}

The subject and population should be the researcher's first point of consideration when developing a 3D hand anthropometric study. By considering the subject and population early, considerations such as forearm length and finger span variability, hand dominance, location of population, and ethnicity can encourage better decision making throughout the process. Because hands are variable between subjects, table 1 outlines several considerations that are important.

Table 1. Subject and population considerations

\begin{tabular}{|l|l|}
\hline Assessment & Importance \\
\hline $\begin{array}{l}\text { Size of subject arm and } \\
\text { hand }\end{array}$ & $\begin{array}{l}\text { Stability frames need to be built to accommodate for subject variability } \\
\text { including forearm length and finger span. } \\
\text { Some scanners, such as foot scanners, are not big enough to enable a } \\
\text { variety of dynamic positions of the hand. }\end{array}$ \\
\hline $\begin{array}{l}\text { Location and recruitment } \\
\text { (technology) }\end{array}$ & $\begin{array}{l}\text { Understanding the target population will allow researchers to make } \\
\text { more educated decisions when selecting technology. } \\
\text { Hand-held and small, mobile scanners provide opportunities to target } \\
\text { specific user-groups and travel to scan diverse populations. }\end{array}$ \\
\hline Ethnicity (skin color) & $\begin{array}{l}\text { Ethnicity will play a role in selecting specific colors of landmarks for color } \\
\text { scanning. } \\
\text { Many scanning technologies have issues capturing dark colors, which } \\
\text { could cause incomplete data in the scans of individuals with darker skin. } \\
\text { It's important to select a scanner that can capture all skin colors under } \\
\text { various lighting conditions. }\end{array}$ \\
\hline
\end{tabular}

\subsection{Technology Selection}

The 3D Hand Scanning Attribute Framework [7] may be used to select the appropriate scanning technology for the research. Based on the subject and population considerations, some technology attributes that are important for selecting a scanning technology include vendor/location, hand-held compatibility, scanner size, weight, envelope, supporting weight, price; along with scanner technology, timing, resolution, color capture, and file saving.

The most important attributes to consider when developing 3D scanning research design are scanning envelope size, length of scan, resolution, and color. The scanning envelope will affect hand positions and platform development. If the scanning envelope is too small, such as some foot scanners, it is difficult to scan the hand in different positions or to ensure that the forearm and entire hand are captured in the scan. If the scanning envelope is too large, the resolution of the scan could be affected and hardto-capture areas such as fingertips and finger crotches might be distorted or not fully visible. Similarly, the resolution capabilities of the scanner are integral to capturing the fine details of the hand such as crease lines. The time it takes for the scanner to take a scan of the hand will play a significant role in planning many of the process steps proposed in this paper. A longer scan time might require the development of stability frames for scanning various hand positions. A faster scan time will enable a more robust collection of hand scans in different positions without adding significant time to the overall scanning process. Resolution and color/texture are important for collecting clear scans that enable the analysis of landmarks, wrinkes/creases, skin color, and non-traditional measurements.

\subsection{Landmark Considerations}

Anthropometric landmarks of the hand ensure the 3D hand scan can be measured accurately post capture. Traditional hand anthropometric studies are missing several key landmarks that are important to designing products and tools for the hands.

New, product-specific landmarking methods were developed by the researchers of this paper. For glove design, the researchers included landmarking crease lines as used by hand surgeons to understand where gloves need to flex, the 14 joint locations of all 5 fingers, finger crotches, tips of fingers, and wrist location (see Figure 4) [5]. The researchers also developed a protocol for landmarking that included notating the palmar and dorsal sides of the hand, as the landmarking locations are different on each plane. 


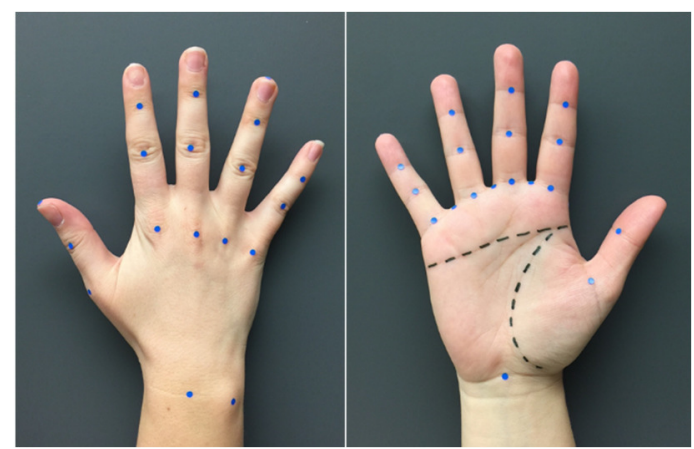

Figure 4. Example of landmarking dorsal and palmar sides of the hand for 3D scanning. [5]

Methods used to landmark hands is dependent upon the scanning technology. Color scanners allow the use of felt tip, washable pens in color and stickers; whereas, non-color scanners require a bit more creativity, such as 3D shapes like pyramids so the scanner can "see" the shape.

For future studies, there is ample opportunity to develop new methods of landmarking the hand to enhance the knowledge of how the hand 'works' in relation to a specific occupation.

\subsection{Hand Scanning Positions}

In the past, only anatomical hand positions were measured for anthropometric data (not splayed or in ergonomic position). Exploration to determine measurements of the functional hand, such as thumb reach for handheld devices and functional grip, is needed for anthropometric studies developed for a specific product. Careful consideration to hand scanning positions will enable researchers to analyze the hand across dynamic positions.

Figure 5 demonstrates several hand positions, such as grasping positions and with fingers splayed, that can be compared across subjects to see how the dimensions of the hand change depending on the position.

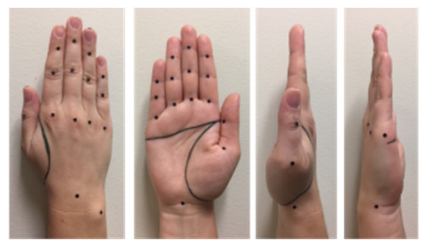

[Position 1]

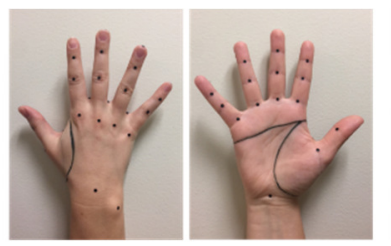

[ Position 2]

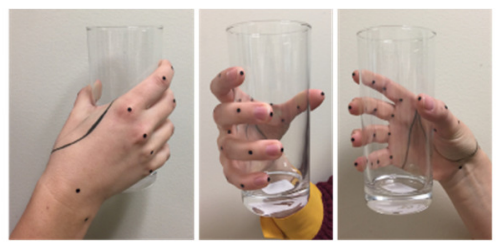

[Position 7]

\section{Hand changes when the finger positions change}

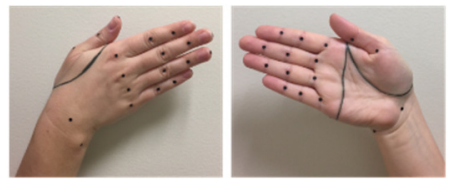

[Position 3]

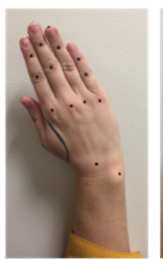

[Position 4]

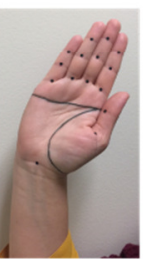

Range of movement for the hand at the most extreme positions

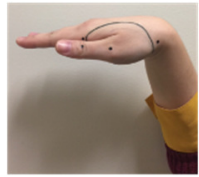

[Position 5] [Position 6]

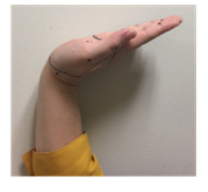

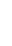

Figure 5. Hand positioning examples. [6]

\subsubsection{Stability frames}

$3 \mathrm{D}$ hand researchers need to consider how the hand is stabilized during scanning. For flatbed foot scanners, the process is simple: the subject places their hand on the bed and the scanning envelope captures the whole hand. With some portable technologies, the hand must be stabilized to limit sway due to the length of the scan. Different methods of stability frames were tested to enable reliable, smooth scans. 
Stability frames need to stabilize the hand, as well as provide 360 degrees of unobstructed views of the hand. The University of Minnesota and Cornell University have collaborated on the creation of several types of frames, including molded Plexiglas frames and frames created from wood, metal, and Plexiglas (as seen in Figure 6 and 7). The key to creating stability frames is to ensure it is mobile, stable, and that it accommodates subject variability including forearm length and finger span. Frames and devices that support the dynamic hand, such as a clear tube or glass that can demonstrate a hand grip (Figure 7), need to be developed and tested to understand hand dimensional change across functional positions.

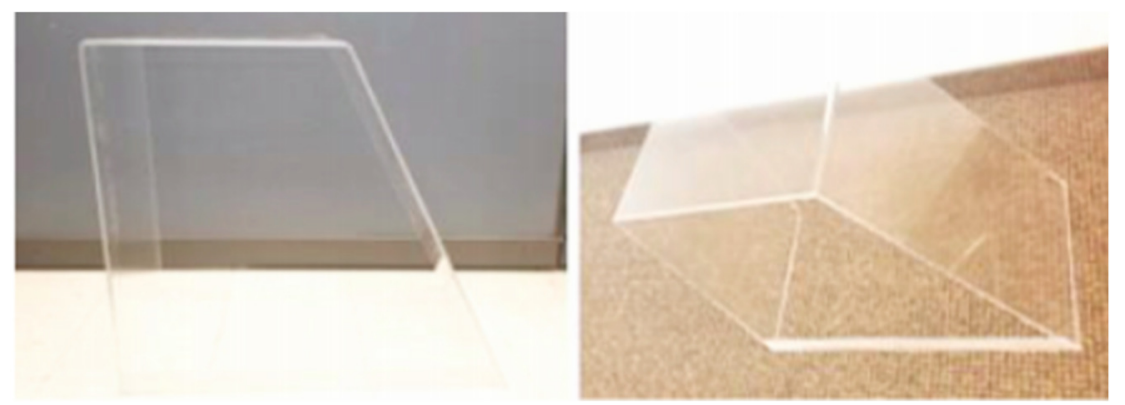

Figure 6. Plexiglas stability frames.
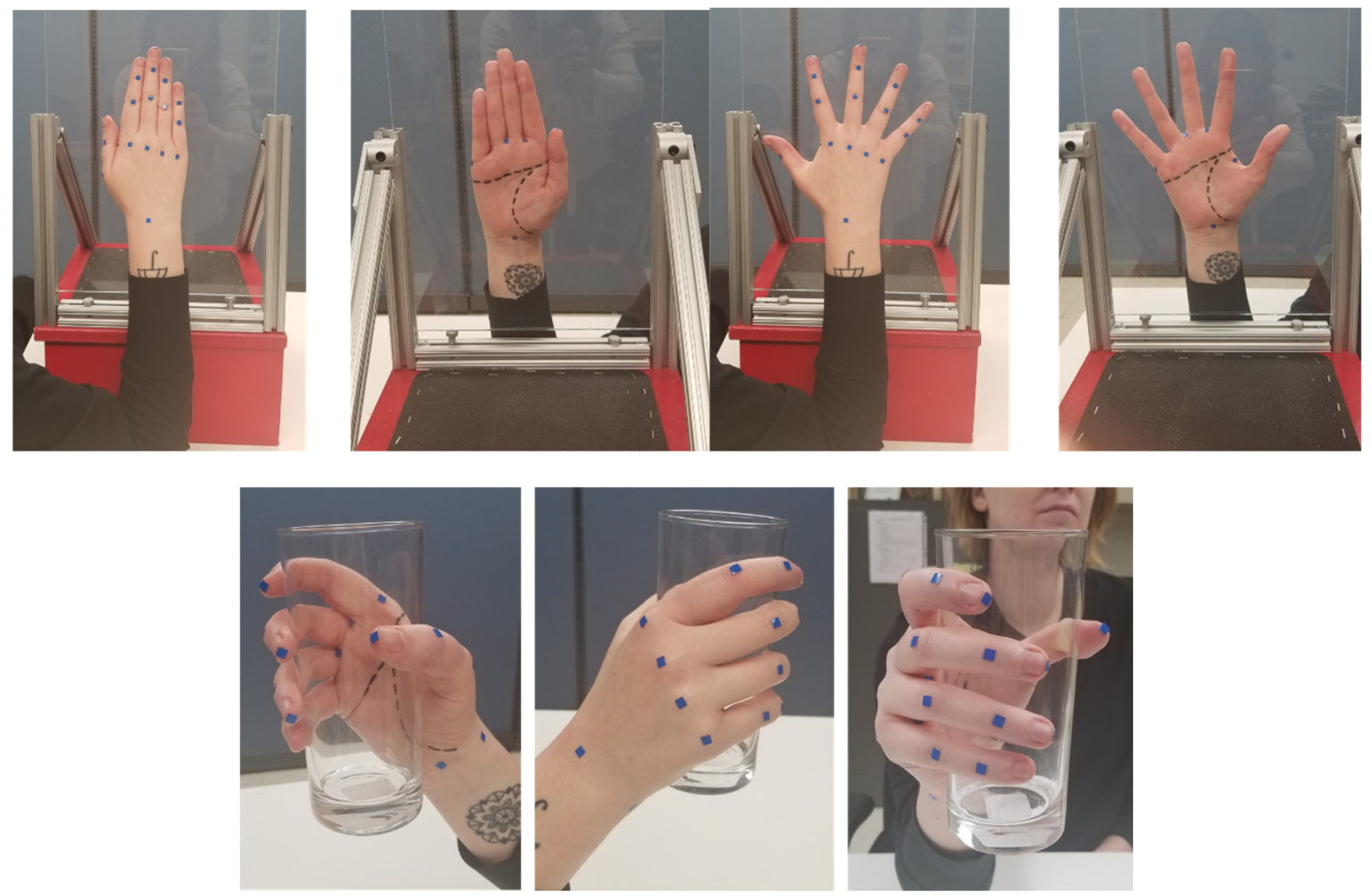

Figure 7. Stability frames and scanning tools.

\subsection{Scanning Process}

Much like traditional anthropometric studies, researchers will need to thoroughly develop a standard operating procedure to ensure the repeatability of the scanning and analysis process. Resources, supplies, and timeframe to conduct the research, training of personnel, creating a procedure that is conscious of subject time constraints, and location/s of scanning are a few considerations important to $3 \mathrm{D}$ hand scanning studies. Without careful planning and communication among the research team, it will be difficult to produce high quality, reliable data. 


\subsection{Scan Analysis}

A number of software platforms exist to measure and analyze scans. 3D hand scan data can be imported into software using a range of file formats, such as .obj and .stl. The researchers of this paper have used AnthroScan, Scanworx, Geomagic, and Meshlab to analyze measurements and features of 3D hand scans.

There are many types of measurements that can be analyzed, including lengths, circumferences and widths (e.g., finger length, palm circumference and hand breadth). The measurements are derived from the landmarks placed on participants prior to scanning, and can be customized. Deriving measurements from landmarks will improve the accuracy of the measurement and measurement error between researchers.

Developing measurements and measurement protocols that are specific to the products that are being developed can improve the end product. For instance, specific anthropometric hand data important for glove design include finger lengths, crotch depths, palm and padding, back of hand length and breadth, and wrist circumference; these measurements can improve dexterity, gripping, hand entry, adduction, abduction, squeezing, etc. in the glove design. Many of these measurements can be programed in the chosen measurement software to produce semi-automatic measurements, saving the researcher significant time.

The measurements can then be compared (univariate, bivariate, multivariate statistics) between all scanned subjects, and subgroups can be created for specific characteristics: women, different ethnic groups, age, occupational type and experience, world regions, etc. Basic statistical methods of mean/median/mode, percentages, probability distribution, and linear regression can be calculated to help understand the data. Shape-based analysis methods, including volumetric measures, crosssections and measurements that relate to specific product parameters (e.g., location of glove flex location on the palm of the hand) offer researchers significant knowledge advancement of hand anthropometry.

\subsection{Visualization techniques during the design process}

Through different visualization techniques, 3D hand scans can allow researchers to understand how specific users' hands interface with new product design, sizing and fit systems. Some of the techniques used during the design process, can include: ideation underlays, comparison of dressed versus undressed user hand scans to understand fit, 3D pattern simulation and fit, and evaluation of how new product concepts (created in 3D CAD) interface with hand scans.

\subsubsection{Design ideation underlays}

The easiest way to utilize 3D scans during the design process, is by using them as underlays for design ideation. The scan file is merely saved in a format (e.g., pdf, jpg, tiff or png), where it can be imported into drawing software (e.g., Illustrator or Photoshop), and then placed in as an underlay for the designer to draw over. Using 3D scans as underlays are quite useful, as they can provide: a realistic view of the hand that can be difficult for designers to draw, correct proportions of specific users, and different views of the hand so the artwork can be drawn at any angle (e.g. $3 / 4$ view).

\subsubsection{Dressed versus undressed user hand scans to understand fit}

3D hand scans along with scans of users' hands while wearing or holding products (e.g.,gloves and medical instruments) can be merged together to compare how a product interfaces and fits. For example, 3D scan data can be collected from subjects with and without wearing PPE gloves, aligned, and merged to evaluate the distribution of gaps (ease) between the body surface and inner side of PPE items as part of fit evaluation (Figure 6) [8]. Wrinkle analysis can also be used to identify areas of stress and need for extra fullness in different hand positions. 


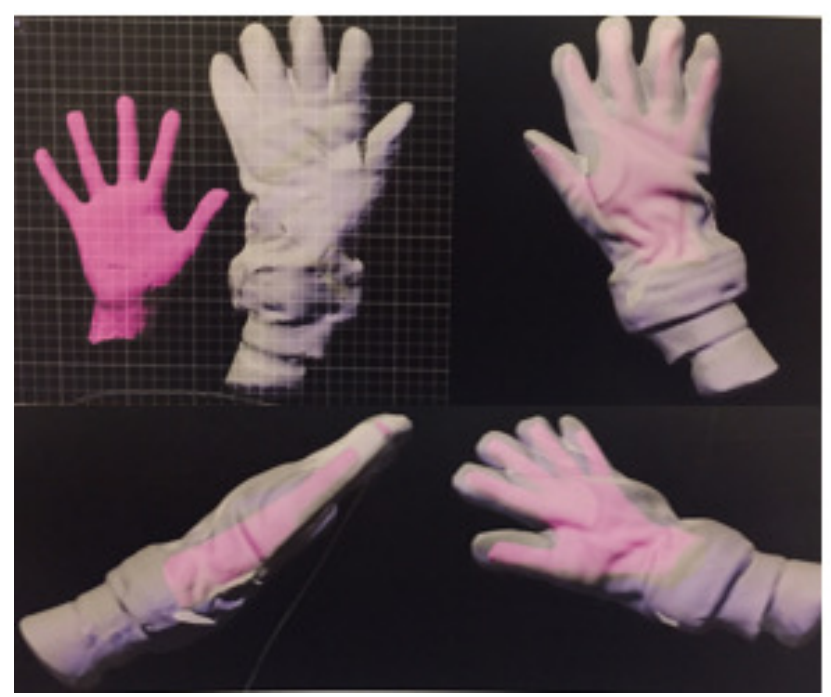

Figure 6. Merging of 3D hand scan data with and without product to see areas in need of fit improvement [8].

\subsubsection{D Pattern simulation and fit}

Another way $3 D$ hand scans can be used to visualize new product design sizing and fit is by importing them into pattern drafting and draping software systems (e.g., OptiTex 3D, Browzwear or CLO3D) to analyze glove pattern fit. The 3D hand scan is simply imported into the software and once the patterns are drafted, they can be stitched together to drape around the hand scan. Researchers can use the pressure map function to see areas of tight and loose fit, and see where the product does not drape appropriately around the hand. From there the design or patterns can be revised to improve fit and function.

\subsubsection{Evaluation of how new 3D product concepts interface with 3D hand scans}

$3 \mathrm{D}$ scans where the hand is posed in a ergonomic/functional position can be used to analyze the design of new tools by inserting 3D files (from Rhino, SolidWorks, etc) to understand the interface of the new design with the user's hand. Information gathered from this visualization can help reduce product development costs by allowing the designer to work digitally before cutting expensive molds.

\section{Conclusion}

As technology improves, our processes for collecting data need to adapt. New 3D scanning technology enables a more robust collection of anthropometric, ergonomic, and design data for the hand.

Future 3D hand anthropometric data and design research will have a profound impact on future glove and tool design for a range of fields and consumers. The application of the 3D hand scanning process considerations model will enable innovative anthropometric and ergonomic research for the hand to occur. Anthropometric and ergonomic data should be integrated with user data to develop new products for hands. Through holistic 3D anthropometric data integration, new hand product design criteria can be established for glove and tool design, as well as new criteria for hand product sizing systems. 3D scans offer researchers and designers the ability to enhance design evaluation, prototypes, and testing of hand products. Furthermore, the anthropometric data, as well as the scans themselves can be used to develop sizing systems for improved fit, size coverage, and effectiveness of performance. Future research includes pooling the results of a larger dynamic, 3D hand data in an effort to provide practical guidance for the design and manufacture of hand products and tools.

\section{Acknowledgement}

This material is based upon work supported by the Minnesota Agricultural Experiment Station, and the National Institute of Food and Agriculture, U.S. Department of Agriculture, Hatch under MIN-53-088. 


\section{References}

[1] Harrison, C.R. and Robinette, K.M., 2002. CAESAR: Summary statistics for the adult population (ages 18-65) of the United States of America. AIR FORCE RESEARCH LAB WRIGHTPATTERSON AFB OH HUMAN EFFECTIVENESS DIRECTORATE.

[2] H. Daanen and F. Ter Haar, "3D whole body scanners revisited", Displays, vol. 34, no. 4, pp. 270275, 2013.

[3] S. Nasir, O. Troynikov and C. Watson, "Skin Deformation Behavior during Hand Movements and their Impact on Functional Sports Glove Design", Procedia Engineering, vol. 112, pp. 92-97, 2015.

[4] L. Griffin, et al., "Dimensions of the Dynamic Hand: Implications for Glove Design, Fit, and Sizing." International Conference on Applied Human Factors and Ergonomics. Springer, Cham, 2018.

[5] L. Griffin, et al., "Methods and Tools for 3D Measurement of Hands and Feet." International Conference on Applied Human Factors and Ergonomics. Springer, Cham, 2018.

[6] Kim, N., et al, "A Three-Dimensional Assessment of Hand Dynamics: A Pilot Study of Seven Hand Positions", Presented at Applied Ergonomics Conference, 2018.

[7] S Sokolowski, et al., "Current Landscape for Collecting Hand Anthropometric Data" In Proc. of 3DBODY.TECH 2018 - 9th Int. Conf. and Exh. on 3D Body Scanning and Processing Technologies, Lugano, Switzerland, 16-17 Oct. 2018

[8] S. P. Ashdown \& J.M. Stull, "Analysis of fit and sizing of firefighter gloves using 3D data", International Conference on Applied Human Factors and Ergonomics, Springer, Cham, 2016. 